\section{Percepción del ambiente educacional en dos escuelas de medicina con currículo tradicional. Estudio longitudinal}

\section{Longitudinal perception of the educational environment in two medical schools with traditional curricula in Chile and Spain}

Background: To establish an educational environment that ensures the quality of the teaching-learning process is a challenge for any educational institution. The questionnaire DREEM (Dundee Ready Educational Environment Measure) is used to identify strengths and weaknesses of an educational environment and to compare different medical schools. Aim: To evaluate the changes in the perception of educational environment by students of the Schools of Medicine of the University of Zaragoza, UZar (Spain) and the University of Chile, UCh (Chile) at two points in their curricula. Material and Methods: DREEM questionnaire was answered by 90 students from the UZar and 87 students of the UCh, when they were in the first year (2009) and in the fourth year of their career (2012). Results: At both universities the overall mean scores of DREAM were significantly higher in students in their first year than those obtained in the fourth year (137.5/118.3 for UZar and 128.6/118.8 for UCh). Items with worse perception in the fourth year were observed in subscales Learning Perception and Atmosphere Perception. Items with good evaluation ( $\geq 3.0)$ were the subscales Perception of teachers, academic self-perception, perception of Environment and Social Self-perception. Conclusions: The perception of Chilean and Spanish students about their educational environments indicates that the stage of their medical training is more important than the geographical context or educational institution.

(Rev Med Chile 2016; 144: 1479-1485)

Key words: Educational Measurement; Education, Medical, Undergraduate; Longitudinal Studies; Surveys and Questionnaires.
'Programa de Farmacología Molecular y Clínica, Instituto de Ciencias Biomédicas, Facultad de Medicina, Universidad de Chile,

Santiago, Chile.

${ }^{2}$ Departamento de Fisiología, Facultad de Medicina, Universidad de Zaragoza, Zaragoza, España.

'Tecnólogo Médico, Magíster

Educación en Ciencias de la Salud

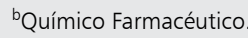

'Médico, PhD.

Declaración de intereses. Los autores declaran que no tienen ningún conflicto de intereses.

Recibido el 30 de marzo de 2016 aceptado el 28 de julio de 2016.

Correspondencia a: Gabriela Díaz-Véliz Programa de Farmacología Molecular y Clínica Instituto de Ciencias Biomédicas Facultad de Medicina, Universidad de Chile. gdiaz@med.uchile.cl
L

os ambientes de aprendizaje han sido destacados como objetivos claves para evaluar los programas de educación médica ${ }^{1}$. Existe consenso entre los educadores médicos que los efectos del entorno educativo son determinantes importantes de las actitudes, conocimientos, habilidades, progresión y conducta de los estudiantes de medicina ${ }^{2,3}$.

Desde 1930 se han desarrollado varios instrumentos para evaluar el entorno de la educación médica, aunque la mayoría de ellos se hicieron obsoletos después de los profundos cambios conceptuales introducidos en los años 80. Entonces se comenzó a hablar de aprendizaje activo, permanente, centrado en el estudiante, basado en problemas, etc. Una herramienta que incorpora la evaluación de la mayoría de estas innovaciones curriculares es el cuestionario DREEM (Dundee Ready Educational Environment Measure) desarrollado por Roff et al. en $1997^{4}$. Ha sido traducido 
y validado en diferentes idiomas, mostrando una alta consistencia interna ${ }^{4-6,9,12}$. La traducción al español fue aplicada por Deza et al en Argentina, encontrando que era confiable, con una consistencia interna de $0,91^{4}$. En Chile fue validado por Riquelme et al en 2009 y luego por Herrera et al, resultando ser altamente confiable, con un Cronbach alpha de 0,91 y 0,92 respectivamente $e^{6,9,12}$.

Este cuestionario DREEM ha sido empleado para conocer diferentes aspectos de la educación médica, identificar fortalezas y debilidades de un entorno educativo ${ }^{3,7}$, comparar diferentes instituciones educativas ${ }^{8,9}$ y evaluar la percepción del impacto de una innovación curricular ${ }^{10-12}$. Los estudios que han utilizado el DREEM para comparar estudiantes en diferentes etapas de su formación informan que aquellos en los primeros cursos se muestran más satisfechos que las cohortes clínicas ${ }^{9,10,13}$. Sin embargo, otras evidencias señalan una satisfacción similar en estudiantes de cursos básicos y clínicos ${ }^{5,14}$. La percepción de los mismos estudiantes durante su formación básica comparada con los cursos preclínicos o clínicos es casi desconocida. El presente trabajo se diseñó como un estudio longitudinal para analizar la percepción del ambiente de aprendizaje de los mismos estudiantes en sus períodos básicos y preclínicos en dos escuelas con diferente ubicación geográfica (España y Chile), ambas con currículos tradicionales similares, basados en disciplinas y enseñanza centrada en el profesor ${ }^{15}$.

\section{Material y Método}

\section{Tipo de estudio y población}

Estudio de tipo cuantitativo, no experimental y longitudinal, centrado en comparar la percepción de estudiantes de medicina acerca del ambiente educacional en dos etapas de sus carreras, en una universidad española y otra chilena. Un total de 177 estudiantes completaron el estudio, siendo evaluados en primer (2009) y cuarto (2012) año de su carrera. De este modo, los mismos 90 estudiantes de la UZar y 87 de la UCh completaron el cuestionario DREEM en ambas ocasiones. A todos ellos se les solicitó su participación voluntaria, después de haber sido informados de los propósitos del estudio. El consentimiento para participar en el estudio se infirió por la realización del cuestionario que fue respondido de manera anónima.

\section{Currículo}

En el período 2009-2012 ambas escuelas de medicina tenían un modelo tradicional de educación médica, con los tres primeros años dedicados a ciencias básicas, y el resto a ciencias preclínicas y clínicas ${ }^{15}$. Las estrategias de enseñanza en los primeros años se basaban, en gran medida, en clases magistrales y otras actividades mayormente centradas en el profesor. En el cuarto año, se combinaba la enseñanza clínica junto al paciente con algunas clases magistrales.

Aunque el año 2010 la UZar adoptó un nuevo currículo basado en el Proceso de Bolonia, los estudiantes encuestados el año 2012 estaban aun bajo el currículo tradicional. El año 2007 se inició un proceso de innovación curricular en la Facultad de Medicina de la Universidad de Chile, pero ésta sólo se concretó en la cohorte del año 2013.

\section{Cuestionario DREEM}

Consta de 50 ítems divididos en 5 subescalas: Percepción del aprendizaje, Percepción de los docentes, Auto-percepción académica, Percepción del ambiente y Auto-percepción social ${ }^{4}$.

Cada ítem se puntúa 0-4 (4= muy de acuerdo, $3=$ de acuerdo, $2=$ inseguro, $1=$ en desacuerdo $y$ $0=$ muy en desacuerdo). Se requiere codificación inversa para 9 ítems negativos. Los resultados se presentan de manera que una mayor puntuación indica un ambiente educativo más favorable. La suma de las puntuaciones de las 5 subescalas entrega una puntuación global máxima de 200 puntos. La interpretación de los puntajes totales y de cada subescala se realizó según lo sugerido por McAleer y Roff ${ }^{16}$.

Con el fin de mostrar las fortalezas y debilidades más específicas dentro del ambiente de aprendizaje, los elementos individuales con una puntuación media $\geq 3$ se tomaron como reflejo de aspectos positivos y se consideraron como áreas de fortaleza; los ítems con una puntuación media $\leq 2$ fueron consideradas como áreas problemáticas.

\section{Análisis estadístico}

Los puntajes DREEM totales y subescalas de cada grupo se expresaron como la media y 95\% de confianza de la media (IC 95\%) y fueron analizados utilizando el GraphPad Prisma v.5. Para determinar diferencias estadísticamente significativas entre grupos se utilizaron las pruebas Kruskal-Wallis y Dunn para comparaciones múltiples 
no paramétricas Las frecuencias de estudiantes se compararon mediante la prueba de chi-cuadrado. Se consideraron diferencias significativas con un $\mathrm{p}<0,05$.

\section{Resultados}

En la muestra de 90 estudiantes de la UZar y 87 de la UCh observamos una edad media de 19,2 $\pm 0,1$ y $19,1 \pm 0,2$ años en 2009 y de $22,5 \pm 0,1$ y $22,1 \pm 0,2$ años en 2012 , respectivamente.

\section{Puntajes totales}

La Tabla 1 muestra que las puntuaciones para los grupos de estudiantes en cuarto año fueron significativamente menores que la de ellos mismos durante su formación básica (2009), en términos de percepción del aprendizaje, percepción del ambiente y percepción global del entorno educativo $(\mathrm{H} 3=70,6, \mathrm{p}<0,0001)$.
Al comparar la frecuencia de estudiantes en cada nivel, observamos que más de $70 \%$ tenía una percepción "más positiva que negativa" sobre el ambiente educativo (Tabla 2). Sin embargo, un número significativamente mayor de estudiantes de cuarto año en la UZar consideró que el ambiente tenía muchos problemas $(21,1 \%$ frente a $0 \%)$. Además, el número de estudiantes que consideraba el medio educativo como excelente disminuyó significativamente $(24,4 \%$ vs $1,1 \%)$. En la UCh no observamos cambios significativos cuando se comparó la percepción de los estudiantes en los años 2009 y 2012.

\section{Puntuaciones en las subescalas o dominios}

2.1. Percepción del aprendizaje: Las puntuaciones obtenidas por los cuatro grupos fueron significativamente diferentes, H3 = 105,70, p < 0,0001, encontrándose las puntuaciones más altas en los estudiantes de primer año (Tabla 1). Las puntuaciones que corresponden a "una percepción más

Tabla 1. Comparación de los puntajes del DREEM (Media e IC 95\%) para los estudiantes de medicina de la Universidad de Zaragoza y la Universidad de Chile (Estudio longitudinal 2009-2012)

\begin{tabular}{|lccccc|}
\hline Subescalas DREEM & $\begin{array}{c}\text { Max } \\
\text { score }\end{array}$ & UZar 2009 & UZar 2012 & UCh 2009 & UCh 2012 \\
\hline 1. Percepción del aprendizaje & 48 & $31,7(30,7-32,6)$ & $21,4(20,2-22,7)^{*}$ & $29,6(28,2-31,1)$ & $24,6(23,1-26,2)^{*}$ \\
\hline 2. Percepción de los docentes & 44 & $26,2(25,1-27,3)$ & $25,1(24,0-26,2)$ & $27,8(26,6-29,0)$ & $27,6(26,1-29,1)$ \\
\hline 3. Auto-percepción académica & 32 & $23,5(22,6-24,3)$ & $22,4(21,5-23,3)$ & $20,8(19,7-21,8)$ & $20,7(19,6-21,9)$ \\
\hline 4. Percepción del ambiente & 48 & $34,6(33,1-36,2)$ & $29,1(27,8-30,4)^{*}$ & $31,3(29,9-32,8)$ & $27,3(25,8-28,9)^{*}$ \\
\hline 5. Auto-percepción social & 28 & $21,5(20,8-22,2)$ & $20,4(19,5-21,2)$ & $19,1(17,3-20,0)$ & $18,5(17,7-19,4)$ \\
\hline Puntaje total del DREEM & 200 & $137,5(134,6-140,4)$ & $118,3(114,0-120,7)^{*}$ & $128,6(124,6-132,7)$ & $118,8(115,1-122,5)^{*}$ \\
\hline Porcentaje del puntaje max & $100 \%$ & $68,8 \%$ & $57,5 \%$ & $64,3 \%$ & $59,3 \%$ \\
\hline
\end{tabular}

${ }^{*} p<0,001$ comparando 2009 (primer año) con 2012 (cuarto año).

Tabla 2. Comparación de los puntajes del DREEM en frecuencia de estudiantes de Universidad de Zaragoza y Universidad de Chile (Estudio longitudinal 2009-2012)

\begin{tabular}{|clcccc|}
\hline & & Universidad de Zaragoza & \multicolumn{2}{c|}{ Universidad de Chile } \\
Puntaje total & Interpretación & $\mathbf{2 0 0 9}$ & $\mathbf{2 0 1 2}$ & $\mathbf{2 0 0 9}$ & $\mathbf{2 0 1 2}$ \\
\hline 50 & Muy pobre & $0 \%$ & $0 \%$ & $0 \%$ & $0 \%$ \\
\hline $51-100$ & Con muchos problemas & $0 \%$ & $21,1 \% *$ & $9,2 \%$ & $11,5 \%$ \\
\hline $101-150$ & Más positivo que negativo & $75,6 \%$ & $77,8 \%$ & $71,3 \%$ & $73,6 \%$ \\
\hline $151-200$ & Excelente & $24,4 \%$ & $1,1 \% *$ & $19,5 \%$ & $14,9 \%$ \\
\hline
\end{tabular}

* $p<0.001$ comparando 2009 (primer año) con 2012 (cuarto año). 
positiva" (31,7 y 29,6) cambiaron a "la enseñanza es vista negativamente" $(21,4$ y 24,6$)$. Esta subescala no obtuvo calificaciones superiores a 3,0 en ningún curso de ambas instituciones. Tres ítems de esta subescala obtuvieron peores puntuaciones en 2012 que en 2009, en ambas universidades (Tabla 4).

2.2. Percepción de los docentes: Para esta subescala no hubo diferencias cuando se compararon los estudiantes de primer y cuarto año en las dos universidades (Tabla 1). Los puntajes oscilaron entre 23 y 33: "moviéndose en la dirección correcta". Los profesores fueron calificados como eficientes y bien preparados para sus clases (media $\geq 3,0$ ), aunque también fueron percibidos como autoritarios (Tablas 3 y 5 ).
2.3. Auto-percepción académica: La percepción general de los estudiantes fue similar los años 2009 y 2012, en ambas universidades (Tabla 1), y los puntajes oscilaron entre 17 y 24 que corresponde a "sentirse más en el lado positivo". La Tabla 3 muestra que todos los estudiantes estaban muy confiados en pasar de curso (puntuaciones medias $\geq 3,0)$.

2.4. Percepción del ambiente: Los estudiantes de primer año de las dos escuelas de medicina tenían una buena percepción sobre el ambiente académico (Tabla 1). Esta percepción disminuyó significativamente tres años más tarde $(\mathrm{H} 3=46.73$, $\mathrm{p}<0,0001)$. Sin embargo, todos los puntajes se encontraron en el rango de 25 a 36, que corresponde a "una actitud más positiva". Las puntuaciones

Tabla 3. Ítems con buena percepción $(\geq 3,0)$ en primer y cuarto año en ambas universidades

\begin{tabular}{|c|c|c|c|c|}
\hline & $\begin{array}{l}\text { UZar } \\
2009\end{array}$ & $\begin{array}{l}\text { UZar } \\
2012\end{array}$ & $\begin{array}{c}\text { UCh } \\
2009\end{array}$ & $\begin{array}{r}\text { UCh } \\
2012\end{array}$ \\
\hline \multicolumn{5}{|l|}{ Ítems de subescala 2: Percepción de los docentes } \\
\hline Los profesores están bien preparados para sus clases & 3,01 & 3,00 & 3,29 & 3,08 \\
\hline Los profesores conocen las materias que dictan & 3,04 & 3,01 & 3,28 & 3,41 \\
\hline \multicolumn{5}{|l|}{ Ítems de subescala 3: Auto-percepción académica } \\
\hline Tengo la confianza de que voy a pasar este año & 3,58 & 3,51 & 3,26 & 3,18 \\
\hline \multicolumn{5}{|l|}{ Ítems de subescala 4: Percepción del ambiente } \\
\hline Siento que puedo hacer todas las preguntas que quiero & 3,07 & 3,03 & 3,13 & 3,15 \\
\hline Me siento cómodo, socialmente, en clases & 3,43 & 3,20 & 3,21 & 3,29 \\
\hline \multicolumn{5}{|l|}{ Ítems de subescala 5: Social Self Perceptions } \\
\hline Tengo buenos amigos en la Escuela & 3,69 & 3,75 & 3,67 & 3,68 \\
\hline Los ambientes físicos de la Escuela son agradables & 3,66 & 3,73 & 3,34 & 3,41 \\
\hline Mi vida social es buena & 3,66 & 3,43 & 3,25 & 3,01 \\
\hline
\end{tabular}

Tabla 4. Ítems con peor percepción $(\leq 3,0)$ en cuarto que en primer año en ambas universidades

\begin{tabular}{|c|c|c|c|c|}
\hline & $\begin{array}{l}\text { UZar } \\
2009\end{array}$ & $\begin{array}{l}\text { UZar } \\
2012\end{array}$ & $\begin{array}{c}\text { UCh } \\
2009\end{array}$ & $\begin{array}{l}\text { UCh } \\
2012\end{array}$ \\
\hline \multicolumn{5}{|l|}{ Ítems de subescala 1: Percepción del aprendizaje } \\
\hline La enseñanza está bien enfocada & 2,64 & 1,28 & 2,55 & 1,86 \\
\hline El tiempo destinado a la enseñanza es bien utilizado & 2,30 & 0,82 & 2,55 & 1,34 \\
\hline La enseñanza está centrada en el estudiante & 2,79 & 1,46 & 2,44 & 1,85 \\
\hline \multicolumn{5}{|l|}{ Ítems de subescala 4: Percepción del ambiente } \\
\hline Los horarios de la Escuela están bien programados & 2,56 & 1,00 & 2,36 & 0,97 \\
\hline El ambiente de la Escuela me motiva a aprender & 3,00 & 2,33 & 2,96 & 2,18 \\
\hline El disfrute de mis estudios en la Escuela pesa más que la tensión que éstos me generan & 2,86 & 2,38 & 2,94 & 1,91 \\
\hline
\end{tabular}


Tabla 5. Ítems con mala percepción $(\leq 3,0)$ en ambos cursos y ambas universidades

\begin{tabular}{|c|c|c|c|c|}
\hline & UZar & UZar & UCh & UCh \\
\hline & 2009 & 2012 & 2009 & 2012 \\
\hline \multicolumn{5}{|l|}{ Ítems de subescala 2: Percepción de los docentes } \\
\hline Los profesores son autoritarios & 2,26 & 2,15 & 2,24 & 2,27 \\
\hline \multicolumn{5}{|l|}{ Ítems de subescala 4: Percepción del ambiente } \\
\hline En la Escuela, la copia en los exámenes constituye un problema & 2,84 & 2,43 & 2,23 & 2,25 \\
\hline \multicolumn{5}{|l|}{ Ítems de subescala 5: Auto-percepción social } \\
\hline Hay un buen sistema de apoyo para los estudiantes que sufren de estrés & 1,26 & 1,39 & 1,49 & 1,3 \\
\hline
\end{tabular}

medias en tres ítems de este dominio fueron más altos para los estudiantes el año 2009 que el año 2012, en ambas universidades. Los estudiantes aseguran que los tiempos en las escuelas no estaban bien programados y que el ambiente escolar no motivaba a aprender. También se consideró que el disfrutar de estar en la escuela fue superado por el estrés que ello significaba (Tabla 4). Sin embargo, los estudiantes de todos los cursos sentían que podían preguntar todo lo que querían y se sentían cómodos en clase (ambos ítems con una puntuación $\geq 3,0$ ) (Tabla 3). Para todos los participantes del estudio la copia en los exámenes fue considerado un problema (Tabla 5).

2.5. Auto-percepción social: No hubo diferencias significativas entre las puntuaciones medias obtenidas en ambas universidades (Tabla 1). Todas las puntuaciones estaban en el rango "No está mal”. Hubo acuerdo general de que todos tenían buenos amigos en sus escuelas, se encontraban notablemente satisfechos con su vida social y sus sitios de estar eran agradables (puntuaciones medias $\geq 3,25$ ). Sin embargo, los estudiantes tenían la percepción de que el sistema de apoyo institucional para los estudiantes que se estresan era deficiente $(\mathrm{p} \leq 1,49)$ (Tabla 5).

\section{Discusión}

El cuestionario DREEM ha sido ampliamente utilizado para evaluar el ambiente educativo en escuelas de medicina ${ }^{3,7-12}$. Los resultados de este estudio señalan que la percepción de los estudiantes de la UZar y de la UCh es muy similar, tanto en el primer como en el cuarto año. Estos resultados concuerdan con estudios realizados en otras escuelas de medicina, los cuales describen ambientes educativos "con aspectos más positivos que negativos" (50 a 75\% de la puntuación total $)^{8-10,16,17}$. Aunque hay escuelas que han obtenido puntuaciones menores que $50 \%{ }^{11,12}$, pensamos que el rango de 50 a $75 \%$ es demasiado amplio considerando las diferencias significativas descritas en la literatura. Incluso en nuestro estudio, a pesar que la percepción cae en los cursos superiores, todas las puntuaciones totales quedaron dentro del mismo rango $(57,5 \%-68,8 \%)$.

En un estudio multicéntrico realizado en seis escuelas de medicina chilenas, excluyendo la UCh, los resultados variaron en el rango de $51,5 \%$ a $64,2 \%$ y se mostró una significativa variabilidad entre estudiantes de tercer, cuarto y quinto año según la universidad de origen ${ }^{9}$. Otro estudio realizado en la Universidad Católica de Chile ${ }^{12}$, para evaluar la percepción acerca de un nuevo currículo, mostró que los puntajes de los estudiantes de tercero a quinto año eran similares a los presentados en este estudio $(63,8 \%)$. Un estudio previo realizado en la UCh también mostró una disminución significativa en la percepción del ambiente educacional entre estudiantes de primer y quinto año, algo no observado en la Universidad Nacional de Cuyo (Argentina) que posee un currículo basado en competencias ${ }^{8}$. Sin embargo, hay que tener en cuenta que todos los estudios mencionados han sido de corte transversal.

Nuestros resultados comprueban que el porcentaje de estudiantes que tienen diferentes percepciones acerca de su entorno educativo varía con su ubicación geográfica. En la UZar aumentó significativamente el porcentaje de alumnos que percibían el entorno educativo como "con muchos problemas" cuando llegaban a cuarto año $(0 \%$ a $21,1 \%$ ), acompañado de una disminución del porcentaje de estudiantes con la percepción de 
"excelente" (24,4\% a 1,1\%). Sin embargo, en la $\mathrm{UCh}$, estas diferencias no fueron significativas, en forma similar a lo observado en otras universidades nacionales 9 .

En cuarto año la percepción resultó ser baja respecto a "El tiempo destinado a la enseñanza es bien utilizado" y "Los horarios de la escuela están bien programados", lo que demuestra que la gestión del tiempo es un problema general, como han manifestado otros autores ${ }^{8,10,12,17}$. Otros ítems que tienen una puntuación más baja en 2012 que en 2009 son "La enseñanza está bien enfocada" y "La enseñanza está centrada en el estudiante". Ellos se correlacionan bien con la baja puntuación de los ítems "El ambiente de la escuela me motiva a aprender" y "Los profesores son autoritarios", y nos dan la idea de que los profesores no han logrado desarrollar estudiantes independientes. La mala gestión del tiempo de enseñanza es indicativa de que las estrategias de aprendizaje no han sido optimizadas por los profesores y subyacen varias críticas a su desempeño, tales como una enseñanza no focalizada, centrada en detalles y pasiva. Algunas de las áreas problemáticas identificadas en este estudio también lo han sido en otras escuelas de medicina, como la Universidad Católica de Chile $^{12}$ y la Universidad de Leeds ${ }^{7}$. Nuestros resultados deberían incentivar a los docentes a aplicar estrategias metodológicas innovadoras más apropiadas para el logro de aprendizajes en un período razonable de tiempo.

Resulta preocupante observar que una muy baja proporción de estudiantes concuerda en la existencia de un buen sistema de apoyo cuando están estresados, lo que coincide con los hallazgos de otros estudios ${ }^{3,7,12}$. Riquelme et $\mathrm{al}^{12}$, informaron una media de 1,16 desde el tercer al quinto año de medicina, mientras que Whittle et al ${ }^{7}$, informaron puntajes tan bajos como los nuestros, al punto que organizaron grupos focales y utilizaron el correo electrónico para solicitar información adicional que les permitiera interpretar las respuestas y considerar posibles medidas remediales. Concluyeron que el problema se debía a falta de conocimiento de los estudiantes acerca de los servicios disponibles en la universidad. Para solucionar este problema, la Universidad de Leeds diseñó carteles orientando a los estudiantes, lo cual fue bien recibido por éstos ${ }^{7}$. En la UZar y en la UCh existen sistemas de apoyo, pero los estudiantes, aparentemente, no saben dónde están o cómo acceder a ellos. Esto también parece ser el caso en Canadá, donde 51\% de los estudiantes respondió estar inseguro o dudoso frente a esta pregunta ${ }^{3}$.

Otro aspecto de advertencia en ambas universidades fue el tema "En la escuela, la copia en los exámenes constituye un problema", lo cual puede considerarse un factor desmotivante para algunos estudiantes. Este problema también ha sido descrito en la literatura ${ }^{9,10-12}$ y debería inducirnos a verificar si el grado de competitividad es exagerado y si el control de las pruebas es el apropiado.

En general, los estudiantes en primer y cuarto año de ambas universidades concordaron en percibir a los profesores como eficientes y bien preparados para sus clases. Además, se sentían satisfechos con su vida social y con sus amigos y tenían confianza en aprobar el año académico, a pesar de los problemas antes señalados.

En conclusión, este estudio permite monitorear problemas en la percepción del ambiente educacional de estudiantes de dos escuelas de medicina geográficamente distantes. Aunque, en general, ambos grupos de estudiantes percibieron el ambiente de aprendizaje como positivo, este estudio también reveló áreas problemáticas. La percepción de los estudiantes chilenos y españoles disminuyó en el cuarto año respecto a la percepción que tenían al inicio de sus estudios. Obviamente, en los pobres resultados en cuarto año podría jugar un papel la dificultad de la transición de la seguridad de la sala de clase (enseñanza básica) hacia la enseñanza clínica, aunque también puede tener un papel la percepción de falta de disponibilidad del tiempo necesario para realizar las tareas con alguna probabilidad de éxito, lo cual puede ser experimentado como una sobrecarga académica a medida que los estudiantes progresan en su educación médica. Los profesores de ambas escuelas de medicina deberían estudiar las deficiencias identificadas en este estudio a fin de mejorar la situación.

Agradecimientos: A todos los estudiantes de la Universidad de Zaragoza y la Universidad de Chile que participaron en este estudio.

\section{Referencias}

1. World Federation for Medical Education (WFME). International standards in medical education: assess- 
ment and accreditation of medical schools' educational programmes. A WFME position paper. Med Educ. 1998; 32 (5): 549-58.

2. Genn JM. AMEE Medical Education Guide No. 23 (Part 1): Curriculum, environment, climate, quality and change in medical education - a unifying perspective. Med Teach 2001; 23(4): 337-44.

3. Till H. Identifying the perceived weaknesses of a new curriculum by means of the Dundee Ready Education Environment Measure (DREEM) Inventory. Med Teach 2004; 26 (1):19-45.

4. Roff S, McAleer S, Harden RM, Al-Qahtani M, Uddin AA, Deza H, et al. Development and Validation of the Dundee Ready Education Environment Measure (DREEM). Med Teach 1997; 19 (4): 295-9.

5. Rotthoff T, Ostapczuk MS, de Brun J, Decking U, Schneider M, Ritz-Timme S. Assessing the learning environment of a faculty: Psychometric validation of the German version of the Dundee Ready Education Environment Measure with students and teachers. Med Teach 2011; 33 (11): e624-36.

6. Herrera C, Padilla O, Solís N, Pizarro M, Kattan E, Díaz LA, et al. Análisis psicométrico del cuestionario DREEM para medir el ambiente de aprendizaje en Chile. Rev Educ Cienc Salud 2015; 12 (2): 134-41.

7. Whittle SR, Whelan B, Murdoch-Eaton DG. DREEM and beyond; studies of the educational environment as a means for its enhancement. Educ Health (Abingdon) 2007; 20 (1): 7.

8. Díaz-Véliz G, Mora S, Bianchi R, Gargiulo PA, Terán C, Gorena D, Lafuente JV, et al. Percepción de los estudiantes de medicina del ambiente educativo en una facultad con currículo tradicional (UCh-Chile) y otra con currículo basado en problemas (UNC-Argentina). Educ Med 2011; 14(1): 27-34.

9. Herrera C, Pacheco J, Rosso F, Cisterna C, Aichele D, Becker $\mathrm{S}$, et al. Evaluación del ambiente educacional pre-clínico en seis Escuelas de Medicina en Chile. Rev Med Chile 2010; 138 (6): 677-84.

10. Díaz-Véliz G, Mora S, Escanero JF. Análisis del ambiente educacional tras la implantación del Plan de Bolonia en la Facultad de Medicina de la Universidad de Zaragoza, España. Comparación con la Facultad de Medicina de la Universidad de Chile. FEM 2013; 16 (3): 167-79.

11. Al-Hazimi A, Zaini R, Al-Hyiani A, Hassan N, Gunaid A, Ponnamperuma G, et al. Educational environment in traditional and innovative medical schools: a study in four undergraduate medical schools. Educ Health 2004; 17 (2): 192-203.

12. Riquelme A, Oporto M, Oporto J, Méndez JI, Viviani $\mathrm{P}$, Salech F, et al. Measuring student'perceptions of the educational climate of the new curriculum at the Pontificia Universidad Católica de Chile: Performance of the Spanish translation of the Dundee Ready Education Environment Measure (DREEM). Educ Health (Abingdon) 2009; 22 (1): 112.

13. Al-Ayed IH, Sheik, SA. Assessment of the educational environment at the College of Medicine of King Saud University, Riyadh. East Mediterr Health J 2008; 14 (4): 953-9.

14. Jiffry MT, McAleer S, Fernando S, Marasinghe RB. Using the DREEM questionnaire to gather baseline information on an evolving medical school in Sri Lanka. Med Teach 2005; 27 (4): 348-52.

15. Escanero JF, Mora S, Arce J, Bianchi R, Díaz-Véliz G, Gargiulo PA, et al. Curricula de medicina en Iberoamérica y España. Cap 3 y 6. Prensas Universitarias de Zaragoza, Zaragoza, España, 2009.

16. McAleer S, Roff S. A practical guide to using the Dundee Ready Education Environment Measure (DREEM). In: Genn JM., ed. AMEE Education Guide No 23. Curriculum, environment, climate, quality and change in medical education: a unifying perspective. Dundee: Association for Medical Education in Europe. 2001; 2933.

17. Al-Naggar RA, Abdulghani M, Osman MT, Al-Kubaisy W, Daher AM, Nor Aripin KN, et al. The Malaysia DREEM: perceptions of medical students about the learning environment in a medical school in Malaysia. Adv Med Educ Pract 2014; 5: 177-84. 